\title{
Infected charcot spine arthropathy
}

\author{
Phani krishna karthik Yelamarthy ${ }^{1} \cdot$ Tarush Rustagi $^{1} \cdot$ Rajat Mahajan $^{1} \cdot$ Vishwajeet Singh $^{1}{ }^{1} \cdot$ Kalidutta Das $^{1}$. \\ Murari Lal Bansal ${ }^{1} \cdot$ Bibhudendu Mohapatra $^{1} \cdot$ Harvinder Singh Chhabra ${ }^{1}$
}

Received: 29 May 2018 / Revised: 10 July 2018 / Accepted: 15 July 2018

(C) International Spinal Cord Society 2018

\begin{abstract}
Background Charcot spinal arthropathy (CSA), a destructive spinal pathology, is seen in patients with impaired sensation. Superimposed infection in the affected spinal segments can lead to a challenge in the diagnosis and management. Spinal cord injury (SCI) is the leading cause of CSA as persons with SCI have significantly impaired sensation. Though infection of the CSA is rare, SCI persons are prone to superimposed infection of the Charcot spine. We report atypical presentations of three cases of CSA with superimposed infection.

Case descriptions A 47-year-old male with complete T7-8 SCI developed symptoms suggestive of infection and CSA. He was managed with a posterior vertebral column resection (PVCR) of T12 and intravenous antibiotics as the intraoperative culture showed the growth of E. coli and Pseudomonas. A 26-year-old male with T12 complete paraplegia, post status post open reduction and internal fixation with subsequent implants removal developed infection and CSA over the pseudoarthrotic lesion with destruction of T12 and L1 vertebrae and an external fistulous track. He was managed with debridement and anterior column T11-L1 reconstruction with a Titanium cage and four-rod pedicle screw stabilization construct. A 25year-old male with complete paraplegia with CSA at L4-S1. He underwent PVCR of L5 and L3-S2 posterior stabilization. The intraoperative culture and histopathology were suggestive of tuberculous infection.

Conclusion Pyogenic or tubercular infection of CSA should be considered as a diagnostic possibility in persons with SCI who are more prone to infections. The management includes aggressive debridement and circumferential fusion along with appropriate medications to control the infection.
\end{abstract}

\section{Introduction}

Charcot spinal arthropathy (CSA), though initially described as a complication of tertiary syphilis (Tabes Dorsalis) infection, is more commonly seen after traumatic spinal cord injury (SCI) [1, 2]. Loss-of-deep pain and proprioception (neuro-traumatic theory), autonomic dysregulation (neuro-vascular theory) after SCI or in the setting of any other pre-existing condition causes progressive inflammatory destruction of the intervertebral joints (intervertebral disc and facet joints) due to repetitive micro-trauma leading to intervertebral fluid collection and malalignment of vertebral column [3]. The affected motion segments are at an increased risk of infection which ranges from 14 to $17 \%$ in

Tarush Rustagi

tarush.rustagi@gmail.com

1 Indian Spinal Injuries center, Sector C, Vasant Kunj, New Delhi, India1 10070 different studies [1, 4]. It is important to identify clinical and radiological features of superimposed infection on CSA early, as the radiological picture is often indistinguishable. There are very few case reports of infected Charcot Spine (ICS) reported to date. In this article, we review three cases of surgically managed ICS.

\section{Case study 1}

A 47-year-old man with paraplegia presented with the back pain in the upper thoracic region, recurrent urinary tract infection (UTI) and 6 months of febrile episodes along with the presence of 10 days of bilateral groin swelling. He had sustained traumatic fractures of the T7 and T8 vertebrae, 27 years prior with paraplegia (ASIA Impairment Scale: AIS A) for which a non-instrumented fusion was performed elsewhere. Present imaging (radiographs, CT and MRI) showed gross destruction of the L1 vertebra and partial destruction of the T12 and L2 vertebrae with the presence of intervertebral fluid at the T12-L1 and L1-L2 segments and bilateral psoas abscess (Fig. 1, Table 1). The hematological 


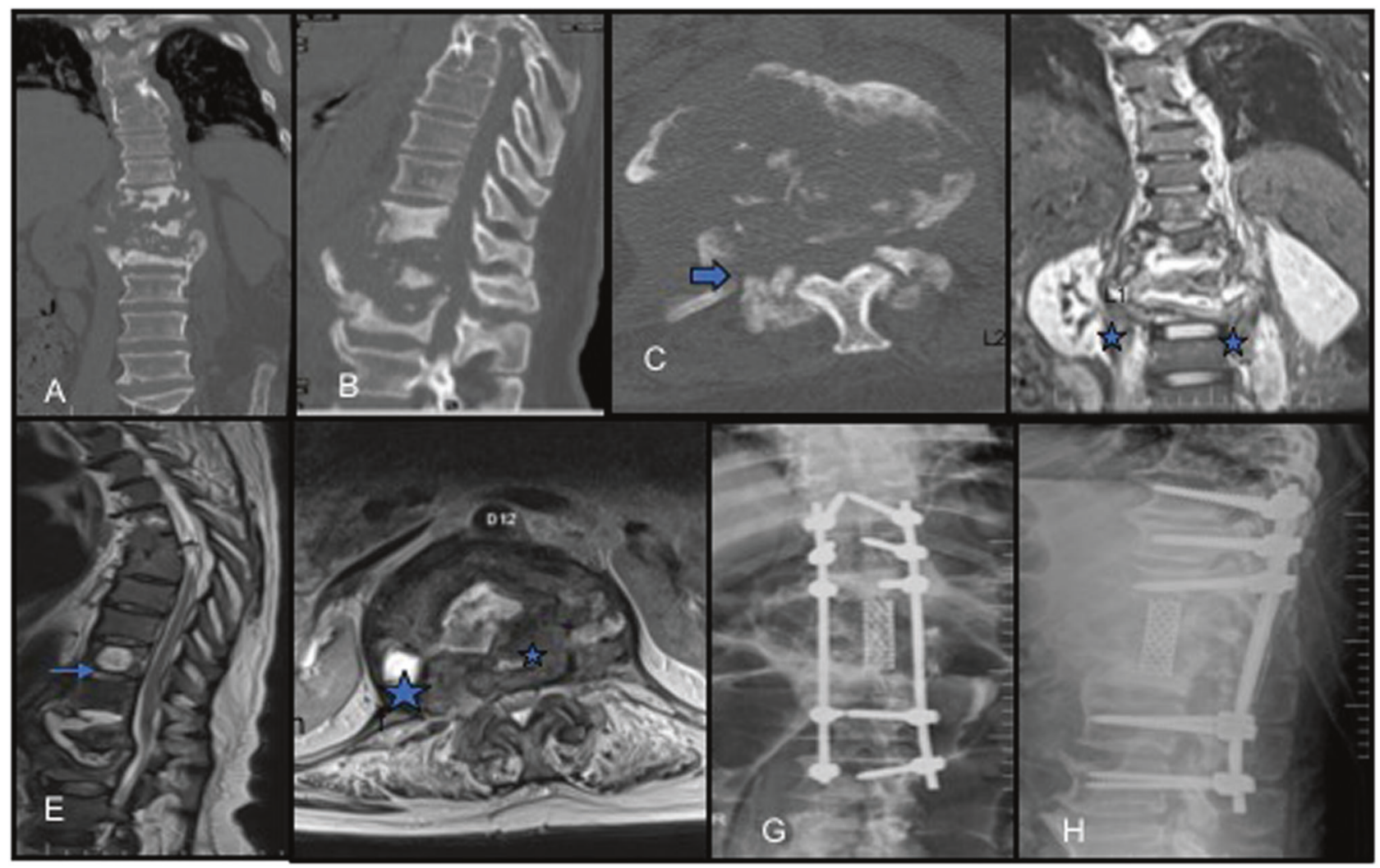

Fig. 1 Preoperative CT $(\mathbf{a}, \mathbf{b}, \mathbf{c})$ and MRI (d, e) showing gross destruction of L1 vertebral body with associated sclerosis in T12 and L2, facet fragmentation (block arrow; image c), psoas abscess ( $\star$;

parameters showed elevated ESR $(125 \mathrm{~mm} / \mathrm{h})$ and CRP (114 mg/L). Suspecting an infection, a percutaneous aspiration of the groin swellings yielded frank pus and showed the growth of E. coli and Pseudomonas on aerobic culture (Table 1). He was then diagnosed with CSA of T12-L2 with secondary infection and was operated by posterior vertebral column resection (PVCR) T12 and L1, anterior column reconstruction (T11-L2) using Titanium mesh cage and posterior stabilization from T9-L3 (Fig. 2). The intraoperative culture also grew E. coli and Pseudomonas and the histopathological examination was negative for malignancy. Postoperatively, based on the sensitivity report, Imipenam was started for 6 weeks and the patient was mobilized. The surgical wound healed uneventfully with the infection resolved both clinically and via laboratory analysis (Blood counts: 5400/ $\mathrm{mm}^{3}$, ESR: $8 \mathrm{~mm} / \mathrm{h}, \mathrm{CRP}$ : $0.5 \mathrm{mg} / \mathrm{L})$ at his last 6 month follow-up.

\section{Case 2}

A 26-year-old male with paraplegia presented with back pain in the anesthetic area and audible clicks at the back and a 9 month history of a bony prominence of the back with sitting. Additionally, he reported a 6-month history of fever image d, f), and intervertebral fluid (small blue arrow; image e). Six month postoperative antero-posterior and lateral radiographs showing a stable construct (Image $\mathbf{g}, \mathbf{h}$ )

and discharging sinuses on the mid-back. He had sustained a fall 6 years prior with T12 vertebral fracture. His neurological status at the time of injury and at presentation was AIS A. As per the records, a posterior stabilization was performed elsewhere. Ten months after the first surgery he developed a deep surgical site infection, which seemed to successfully resolve with implant removal and he was gradually mobilized upon resolution of pain at the surgical site. His present imaging showed partial destruction of the T11 and T12 vertebrae, with intervertebral fluid tracking along the fistulous track along with the presence of syrinx at the T7 vertebral level (Fig. 3, Table 1). Laboratory analysis revealed leukocytosis with elevated ESR $(120 \mathrm{~mm} / \mathrm{h})$ and CRP (169 mg/L) and swabs from the fistulous track showed growth of methicillin-resistant Staphylococcus aureus (MRSA) (Table 1). He was diagnosed with infected CSA: T11-T12 (developed at the site of pseudo-arthrosis of T12) and was managed with a PVCR of T12, anterior column reconstruction (T11-L1) using a Titanium mesh cage and posterior stabilization and fusion using a four-rod construct (Fig. 4). The intraoperative culture showed the growth of MRSA and the histopathological examination was negative for malignancy. Following the culture report, he was treated with intravenous Clindamycin for 6 weeks following which 
Table 1 Radiological/ hematology, microbiology, and pathology features of the cases. Facet joint involvement

\begin{tabular}{|c|c|c|c|}
\hline Radiological feature & Case 1 & Case 2 & Case 3 \\
\hline Location & T12-L1-L2 & $\mathrm{T} 11-\mathrm{T} 12$ & L4-L5-S1 \\
\hline End plate involvement & + & + & + \\
\hline Gas in the disc & - (fluid + ) & $-($ fluid + ) & - (fluid + ) \\
\hline Facet fragmentation + & Facet fragmentation + & Facet fragmentation + & \\
\hline $\begin{array}{l}\text { Joint disorganization } \\
\text { and deformity }\end{array}$ & + & + & + \\
\hline Debris & + & + & + \\
\hline Bony sclerosis & + & + & - \\
\hline $\begin{array}{l}\text { Para spinal soft tissue } \\
\text { component }\end{array}$ & + & + & + \\
\hline DISH & + & - & - \\
\hline Fistula & - & + & - \\
\hline Syrinx & + & + & - \\
\hline Epidural soft tissue & + & + & + \\
\hline $\begin{array}{l}\text { Total leukocyte count } \\
\text { (TLC) }\end{array}$ & 4200 cells/cubic mm & 10,180 cells/cubic $\mathrm{mm}$ & 10,500 cells/cubic $\mathrm{mm}$ \\
\hline ESR & $125 \mathrm{~mm} / \mathrm{h}$ & $120 \mathrm{~mm} / \mathrm{h}$ & $99 \mathrm{~mm} / \mathrm{h}$ \\
\hline CRP & $114 \mathrm{mg} / \mathrm{L}$ & $169 \mathrm{mg} / \mathrm{L}$ & $155 \mathrm{mg} / \mathrm{L}$ \\
\hline $\begin{array}{l}\text { Preoperative urine } \\
\text { culture }\end{array}$ & E. coli & - & - \\
\hline $\begin{array}{l}\text { Preoperative wound } \\
\text { swab/ swelling } \\
\text { aspiration }\end{array}$ & $\begin{array}{l}\text { E. coli and } \\
\text { Pseudomonas (groin } \\
\text { aspirate) }\end{array}$ & $\begin{array}{l}\text { MRSA (swab from the } \\
\text { fistulous track) }\end{array}$ & - \\
\hline $\begin{array}{l}\text { Intraoperative sample } \\
\text { culture analysis }\end{array}$ & $\begin{array}{l}\text { Pseudomonas and } E \text {. } \\
\text { coli }\end{array}$ & MRSA & $\begin{array}{l}\text { Mycobacterium } \\
\text { Tuberculosis }\end{array}$ \\
\hline Histopathology & $\begin{array}{l}\text { Negative for malignancy } \\
\text { and granulomatous } \\
\text { infection }\end{array}$ & $\begin{array}{l}\text { Negative for malignancy } \\
\text { and granulomatous } \\
\text { infection }\end{array}$ & $\begin{array}{l}\text { Suggestive of } \\
\text { granulomatous infection } \\
\text { but negative for malignancy }\end{array}$ \\
\hline
\end{tabular}

$T$ thoracic, $L$ lumbar, $D I S H$ diffuse idiopathic skeletal hyperostosis, $E S R$ erythrocyte sedimentation rate, $C R P$ $\mathrm{C}$ reactive protein, $M R S A$ methicillin-resistant Staphylococcus aureus the infection appeared healed and the surgical wound had healed completely. At his last 1 year follow-up, he had maintained independent sitting balance and was clinically free from spine infection.

\section{Case 3}

A 25-year-old man with paraplegia presented with lower back pain along with audible clicks from the low back with a change of posture and a 6 month history of sitting imbalance. He had a 7-year history of traumatic T12 fracture with resultant AIS A paraplegia for which T10-L2 stabilization and circumferential fusion was performed. His present imaging showed complete destruction of the L5 vertebra and intervertebral fluid (Fig. 5, Table 1). He was afebrile but his laboratory analysis showed elevated ESR $(99 \mathrm{~mm} / \mathrm{h})$ and CRP $(155 \mathrm{mg} / \mathrm{L})$ (Table 1$)$. He was diagnosed with CSA and was managed with L3-S2 posterior stabilization and L4-S1 anterior reconstruction with a Titanium mesh cage (Fig. 5). The intraoperative culture grew Mycobacterium Tuberculosis and histopathological examination was suggestive of granulomatous infection. Postoperatively he was mobilized and treated with 2 months of isoniazid, rifampicin, ethambutol, and pyrazinamide for the mycobacterium followed by a maintenance phase of 10 months using isoniazid and rifampicin. At his 1 year follow-up, the individual was clinically asymptomatic and undergoing rehabilitation.

\section{Discussion}

Charcot arthropathy describes a clinic-radiological pathology with aggressive tissue destruction due to cyclic instability from decreased or absent afferent sensory pathways [5]. Infection in this destructive mess may be underlying or concomitant and at times difficult to distinguish. Clinical features of CSA can include paradoxical back pain in the insensitive area, spinal deformity, sagittal imbalance, audible clicks, and autonomic dysreflexia. Various aspects of these signs were seen in our cases. The radiological findings depend on the stage of presentation of CSA (early 


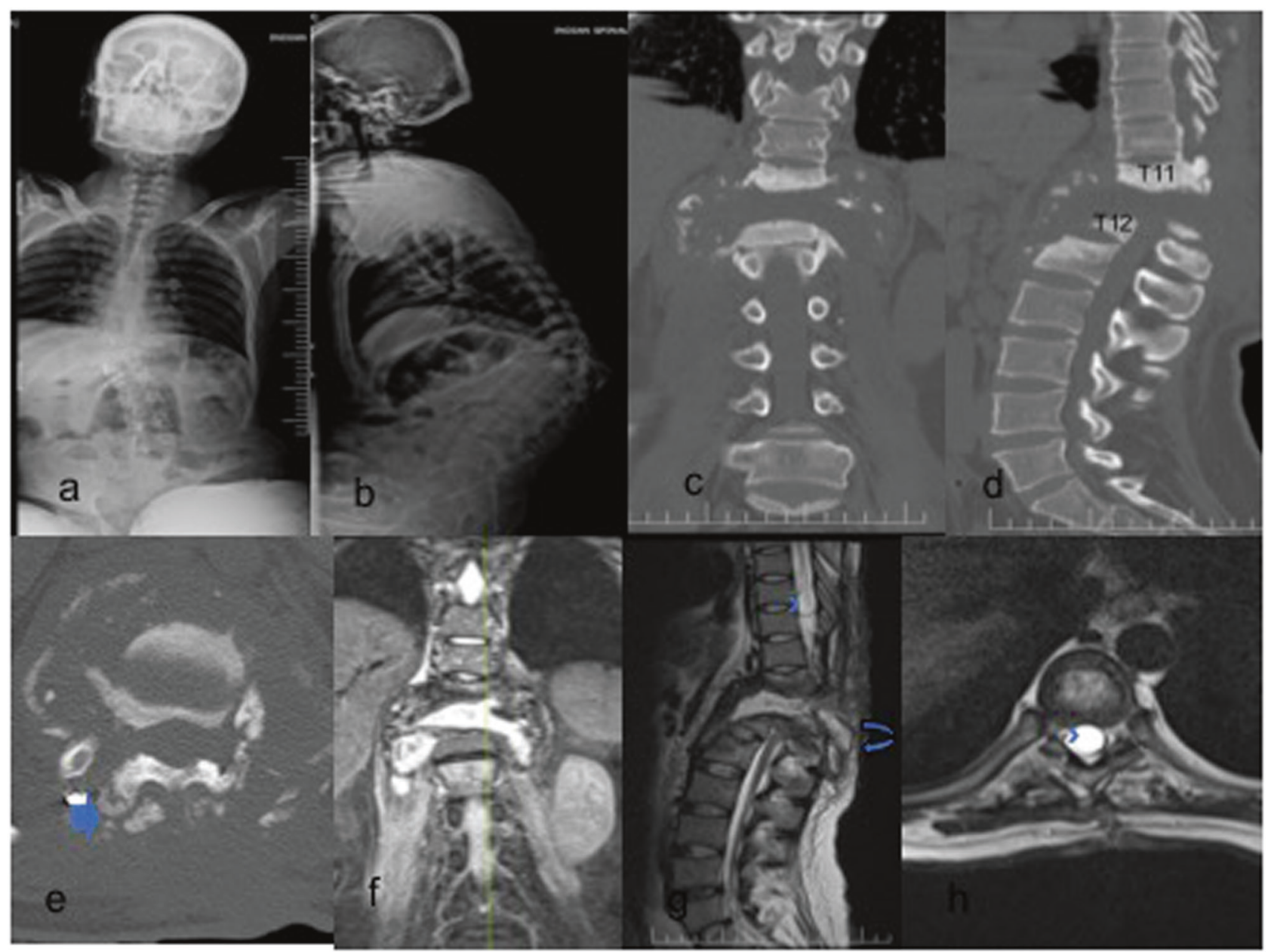

Fig. 2 Preoperative radiographs $(\mathbf{a}, \mathbf{b}), \mathrm{CT}(\mathbf{c}, \mathbf{d}, \mathbf{e})$, and MRI $(\mathbf{f}, \mathbf{g}, \mathbf{h})$ showing partial destruction of T11 and T12 vertebrae with associated sclerosis, facet fragmentation (block arrow; image e), fistulous track (curved arrow; image g), and syrinx (small arrow, image h)

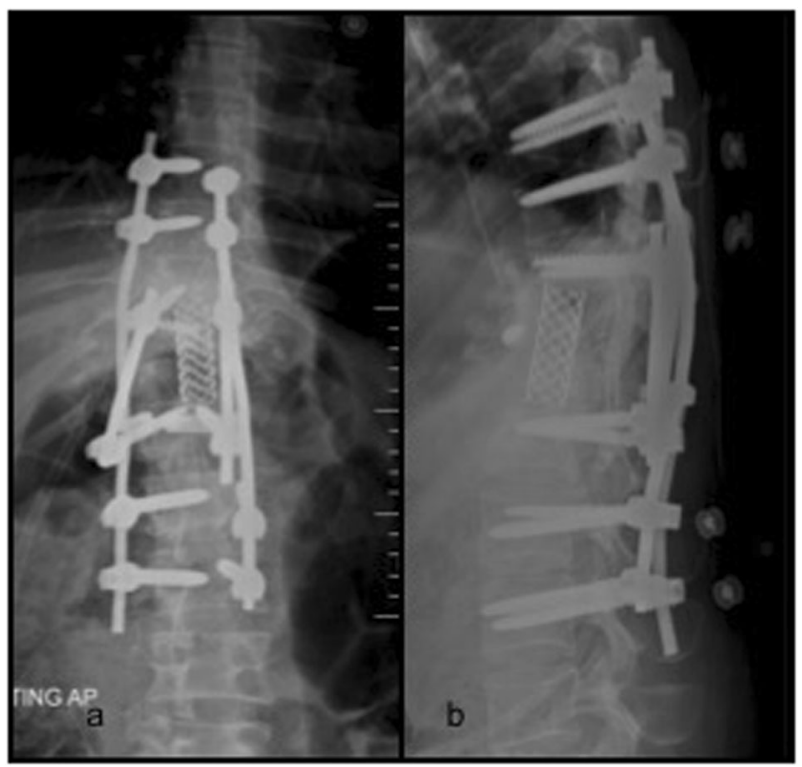

Fig. 3 One year postoperative antero-posterior and lateral radiographs with four rods and anterior mesh cage or late). The early atrophic stage is characterized by the presence of non-specific inflammatory changes while the late hypertrophic stage is characterized by the classical six D's (distension, increased density, destruction, debris, disorganization, and dislocation) seen in Charcot's arthropathy [3]. Intra-discal gas and intervertebral fluid is proportionate to the degree of instability of the involved spinal segment $[6,7]$.

The common differential diagnosis includes spondylodiscitis (pyogenic or granulomatous), spinal tumor, and pseudo-arthrosis. Even though a careful clinicoradiological examination aids in ruling out these alternate diagnoses, isolation may be difficult especially when they co-exist with CSA (Table 2) [1]. This is a possible situation in SCI cases who are at high risk of systemic infection from recurrent UTIs, decubiti, and a dysregulated immune system $[7,8]$. Though, it is not possible to find the geographical distribution of cases of infected CSA, it may have a higher prevalence in developing countries. 


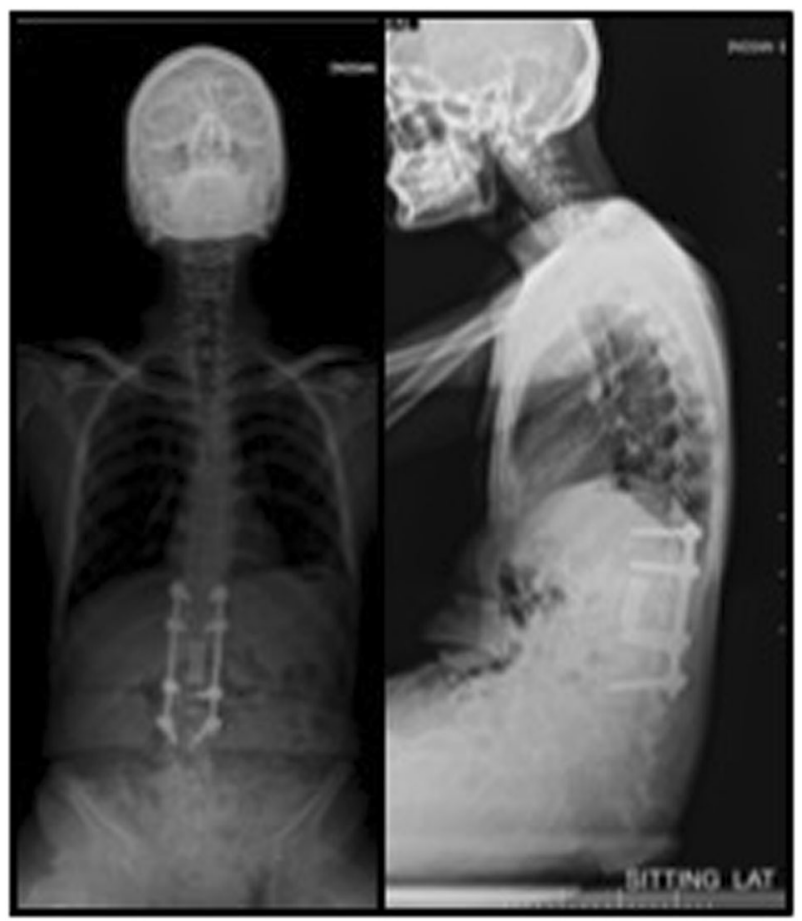

Fig. 4 Preoperative radiographs (a, b), showing previous T11-L3 fusion with destruction of the lumbo-sacral junction

In our series, all three individuals had febrile episodes (except in case 3), elevated acute phase reactants (ESR, CRP) and a positive culture of the intraoperative tissue/fluid (Table 1), which suggested the presence of superimposed infection. We believe the presence of Mycobacterium in the third case, which is a low virulence organism resulted in a lack of strong immune response with resultant absence of fever. Jacobs et al. in their series found ESR to be an inconsistent parameter to determine infection and found CRP to be much more specific [4].

The largest documented case series on CSA by Jacobs et al., and Aebli et al., did not provide the details of their infected CSA cases [4, 9]. We did, however, find case reports documenting the management of infected CSA (Table 3) [7, 10-12]. The source of infection in CSA is commonly through a fistulous tract or an infected decubitus but can also be hematogenous [7]. MRSA was the most commonly reported infection arising from the fistulous track. The probable source of infection in our cases was hematogenous (cases 1 and 3) and through the fistulous skin track in case 2 [7]. Granulomatous infections have rarely been reported in neuropathic joints [13]. We were unable to find any report of tuberculosis infection in CSA. The duration of antibiotic and the route of administration is a matter of debate and no clear consensus is available for chronic spinal infections [13]. The reports mentioning management of ICS do not mention the details of the antibiotic regimen used (Table 3). We preferred to give

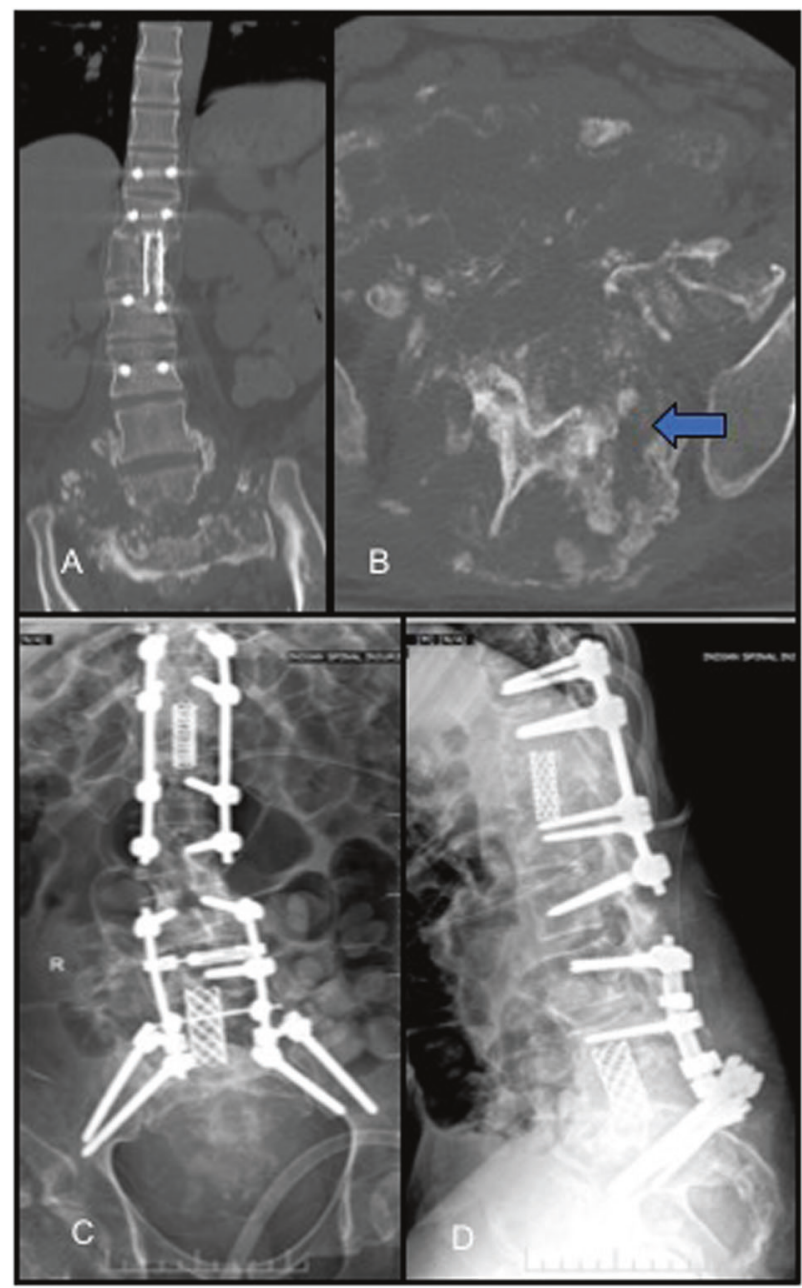

Fig. 5 CT scan showing (coronal and axial images-Image a, b) showing near total destruction of L5 vertebra with facet fragmentation (block arrow; image b) but no accompanying sclerosis. Postoperative antero-posterior and lateral radiographs (Image c, d)

parenteral antibiotics based on the sensitivity for a period of 6 weeks and did not continue with a further oral regimen. With this protocol, we did not see any recurrences. In case 3 , anti-tuberculous medication was given for a prolonged period ( 1 year). The uniqueness of case 3 is the occurrence of a secondary tuberculosis infection in CSA. Even though it is difficult to say whether it was a primary tubercular infection or a secondary, the complete destruction of the L5 vertebra (anterior and posterior elements) and excessive bone debris suggest the diagnosis of CSA. On the contrary, however, the absence of sclerosis of the vertebrae of the involved spinal segment suggests the active destruction by the tuberculous infection.

The usual duration between SCI and diagnosis of CSA documented in literature is around $17 \pm 10$ years, though it varies between 1.5 and 42 years $[1,4]$. In our cases 2 and 3 , time span between the diagnosis of CSA and infection was short (7 years). This short time span was also seen in the 


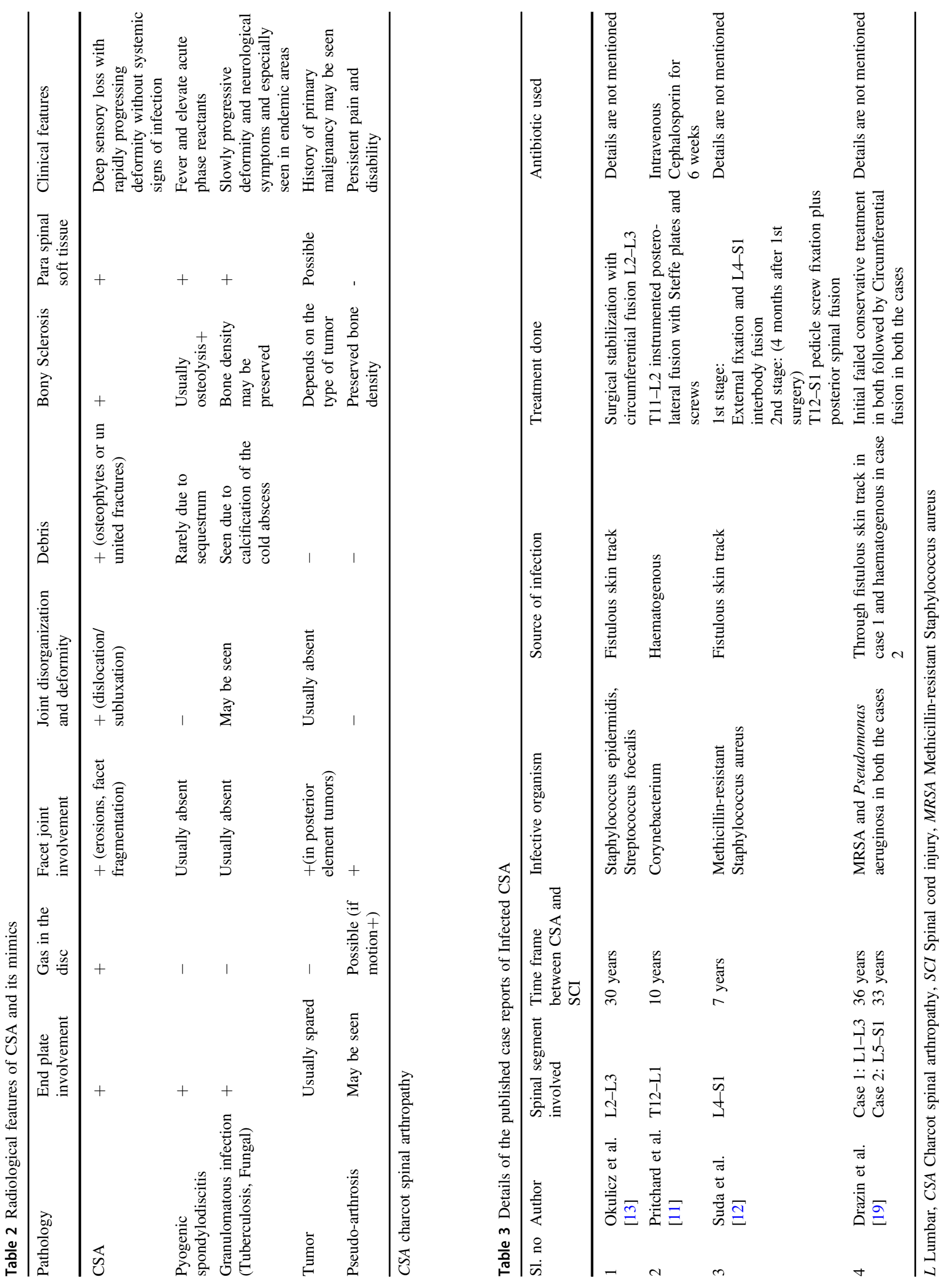


report by Suda et al. [12]. The time span is possibly related to the virulence of the organism, host immunity and the severity of neuropathic destruction. Presence of an overlying skin breakdown or a fistula makes the infection apparent and may be picked up early $[11,12]$. An associated syrinx in case 2 may also have contributed to the relatively early presentation [14].

There is always an apprehension to operate in an infective bed, including the fear of placing metallic implants. However, in ICS, it is imperative to have a stable surgical environment, both to stop the destructive process and to control the infection $[4,9]$. Mere debridement with antibiotics is a suboptimum strategy for controlling infection. The destructive bed acts as a nidus for infection that needs to be removed surgically along with a stable spinal column. This premise was followed in our series where despite evident infection, the spine was thoroughly debrided and stabilized circumferentially.

All the reported cases in the literature were managed surgically by an instrumented circumferential fusion except in one case report where an instrumented postero-lateral fusion was done. Suda et al. used an external fixation in the first stage, which was followed by a definitive internal fixation four months after the first surgery [12].

Numerous surgical strategies have been suggested to deal with the often-complex reconstruction of these neuropathic joints. Jacobs et al. recommended use of rigid fixation to the pelvis, using four rods and suggested used of BMP along with aggressive surgical debridement [4]. Use of quadruple rods have been found to be biomechanically superior for complex spinal reconstructions $[15,16]$. We used the fourrod technique in case 2. The use of BMP, not only enhances fusion but it also has been found to be useful for vertebral pyogenic infections $[17,18]$. We did not use BMP in any of the cases mainly because of cost constraints.

Through our series, we highlight to keep a high index of suspicion for infections in CSA patients where the presentation may be delayed. The infection should be aggressively treated with debridement and rigid stabilization. We also report an unusual case of tuberculous granulomatous infection in CSA, which has not been reported before and its management.

\section{Conclusion}

Pyogenic or tubercular infection of CSA, although a rare occurrence should be considered as diagnostic possibilities, especially in SCI patients who are more prone to infections. Management includes aggressive debridement and circumferential fusion along with appropriate medical management to control the infection.

\section{Compliance with ethical standards}

Conflict of interest The authors declare that they have no conflict of interest.

\section{References}

1. Barrey C, Massourides H, Cotton F, Perrin G, Rode G. Charcot spine: two new case reports and a systematic review of 109 clinical cases from the literature. Ann Phys Rehabil Med. 2010;53:200-20.

2. Moreau S, Lonjon G, Jameson R, Judet T, de Loubresse CG. Do all charcot spine require surgery? Orthop Traumatol Surg Res. 2014;100:779-84.

3. Ledbetter LN, Salzman KL, Sanders RK, Shah LM. Spinal neuroarthropathy: pathophysiology, clinical and imaging features, and differential diagnosis. Radiographics. 2016;36:783-99.

4. Jacobs WB, Bransford RJ, Bellabarba C, Chapman JR. Surgical management of Charcot spinal arthropathy: a single-center retrospective series highlighting the evolution of management. J Neurosurg Spine. 2012;17:422-31.

5. Capps E, Linnau KF, Crane DA. Beyond broken spines-what the radiologist needs to know about late complications of spinal cord injury. Insights Imaging. 2015;6:111-22.

6. D'Anastasi M, Birkenmaier C, Schmidt GP, Wegener B, Reiser MF, Baur-Melnyk A. Correlation between vacuum phenomenon on CT and fluid on MRI in degenerative disks. Am J Roentgenol. 2011;197:1182-9.

7. Morita M, Miyauchi A, Okuda S, Oda T, Yamamoto T, Iwasaki M. Charcot spinal disease after spinal cord injury. J Neurosurg Spine. 2008;9:419-26.

8. Cruse JM, Keith JC, Bryant ML, Lewis RE. Immune systemneuroendocrine dysregulation in spinal cord injury. Immunol Res. 1996; 15:306.

9. Aebli N, Pötzel T, Krebs J. Characteristics and surgical management of neuropathic (Charcot) spinal arthropathy after spinal cord injury. Spine J. 2014;14:884-91.

10. Mikawa Y, Watanabe R, Yamano Y, Morn S. Infected Charcot spine following spinal cord injury. Spine. 1989;14:892-5.

11. Pritchard JC, Coscia MF. Infection of a Charcot spine. A case report. Spine. 1993;18:764-7.

12. Suda $Y$, Saito $M$, Shioda $M$, Kato H, Shibasaki K. Infected charcot spine. Spinal Cord. 2005;43:256.

13. Okulicz JF, Lloyd BA, Krause JO, Conger NG. Mycobacterium tuberculosis infection of a presumed Charcot joint. South Med J. 2005;98:924-6.

14. Grassner L, Geuther M, Mach O, Bühren V, Vastmans J, Maier D. Charcot spinal arthropathy: an increasing long-term sequel after spinal cord injury with no straightforward management. Spinal Cord Ser Cases. 2015;1:15022.

15. Shen FH, Harper M, Foster WC, Marks I, Arlet V. A novel "fourrod technique" for lumbo-pelvic reconstruction: theory and technical considerations. Spine. 2006;31:1395-401.

16. Shen FH, Qureshi R, Tyger R, Lehman R, Singla A, Shimer A, et al. Use of the "Dual Construct" for the management of complex spinal reconstructions. Spine J. 2018;18:482-90.

17. Allen RT, Lee YP, Stimson E, Garfin SR. Bone morphogenetic protein-2 (BMP-2) in the treatment of pyogenic vertebral osteomyelitis. Spine. 2007;32:2996-3006.

18. Drazin D, Choi E, Garcia A, Rustagi T. Bone morphogenic proteins are a good choice for select spinal surgeries and merit further research. J Spine Surg. 2017;3:119.

19. Conterno LO, da Silva, Filho CR. Antibiotics for treating chronic osteomyelitis in adults. Cochrane Database Syst Rev. 2009;3: CD004439. 